\title{
Greater Occipital Nerve Block for Acute Treatment of Migraine Headache: A Large Retrospective Cohort Study
}

\author{
Sorcha M. Allen, MB BCh, BAO, Farouk Mookadam, MB BCh, MSc, \\ Stephen S. Cha, MS, John A. Freeman, DO, Amaal J. Starling, MD, and \\ Martina Mookadam, MD
}

Introduction: Greater occipital nerve (GON) blocks are frequently used to treat migraine headaches, although a paucity of supporting clinical evidence exists. The objective of this study was to assess the efficacy of GON block in acute treatment of migraine headache, with a focus on pain relief.

Methods: This retrospective cohort study was undertaken between January 2009 and August 2014 and included patients who underwent at least $1 \mathrm{GON}$ block and attended at least 1 follow-up appointment. Change in the 11-point numeric pain rating scale (NPRS) was used to assess the response to GON block. Response was defined as "minimal" (<30\% NPRS point reduction), "moderate" (31-50\% NPRS point reduction), or "significant" (>50\% NPRS point reduction).

Results: A total of 562 patients met inclusion criteria; 423 were women (75\%). Mean age was $58.6 \pm$ 16.7 years. Of these 562,459 patients $(82 \%)$ rated their response to GON block as moderate or significant. No statistically significant relationship existed between previous treatment regimens and response to GON block. GON block was equally effective across the different age and sex groups.

Conclusions: Greater occipital block seems to be an effective option for acute management of migraine headache, with promising reductions in pain scores. (J Am Board Fam Med 2018;31:211-218.)

Keywords: Migraine Headache, Nerve Block, Pain, Retrospective Studies, Treatment Outcome

Treatment of migraine headache is an evolving area of interest among headache specialists. A variety of medications with a spectrum of action are used in both acute and prophylactic management. Guidelines from the American Academy of Neurology and the American Headache Society (2012) are available to help direct care. ${ }^{1}$ Rescue medications for acute migraine typically consist of nonsteroidal anti-inflammatory medications, acetaminophen,

This article was externally peer reviewed.

Submitted 6 May 2017; revised 12 October 2017; accepted 17 October 2017.

From the Departments of Internal Medicine (SMA), Family Medicine (MM), and Neurology (AJS); and the Divisions of Biomedical Statistics and Informatics (SSC), Anaesthesiology, Physical Medicine and Rehabilitation (JAF), and Cardiovascular Diseases (FM), Mayo Clinic, Phoenix, AZ.

Funding: none.

Conflict of interest: AJS has received consulting fees from Amgen and eNeura.

Corresponding author: Martina Mookadam, MD, Mayo Clinic, 13737 North 92nd St, Scottsdale, AZ 85260 (E-mail: mookadam.martina@mayo.edu). and triptans; early administration yields the most promising symptom improvement. Preventive medication classes include antiepileptics, antihypertensives, and antidepressants, along with nonpharmacologic therapies such as acupuncture and physical therapy. ${ }^{2}$ Despite all these available treatments, many patients continue to experience poor symptom control.

The International Classification of Headache disorders, third edition (ICHD-3; beta version) ${ }^{3}$ divides migraine into 2 major subtypes: with and without aura. Studies suggest that a peripheral mechanism may contribute to the symptoms of migraine-a theory that the literature reinforces through demonstrations of improvement in migraine headaches following injection of the botulinum A toxin. ${ }^{4,5}$ The greater occipital nerve (GON) originates in the dorsal ramus of the $\mathrm{C}_{2}$ as well as the $\mathrm{C}_{3}$ segments of the spinal cord and comprises sensory fibers alone. Its sensory distribution pathway includes the posterior part of the head and 
extends anteriorly toward the vertex, becoming superficial at the inferolateral aspect of the occipital protuberance. It is thought that sensory input from the GON and the ophthalmic branch of the trigeminal nerve converges into the trigeminal nucleus caudalis, which is hypothesized to be the reason why occipital neuralgia is sometimes associated with migraine headache symptomatology. ${ }^{6}$ GON block decreases afferent input to the trigeminal nucleus caudalis, resulting in central pain modulation and reducing neuronal hyperexcitability at the level of second-order neurons. ${ }^{7}$ The procedure to inject the GON is demonstrated in Figure 1; this involves inserting a needle into the inferolateral aspect of the occipital protuberance and injecting a combination of local anesthetic and a corticosteroid.

No current guidelines include GON block in the management of migraine headache ${ }^{1,8}$, although many studies have researched this, with variable results and conclusions., ${ }^{7-19}$ Four randomized, placebo-controlled trials examining the use of GON block in the treatment of migraine show equivocal results. ${ }^{16-19}$ The most recent of these studies showed promise. ${ }^{19} \mathrm{We}$ undertook this retrospective cohort study to assess the efficacy of GON block in the acute treatment of migraine headache, having anecdotally experienced good results in our clinical practice.

\section{Figure 1. Illustration demonstrating the occipital nerve block procedure.}

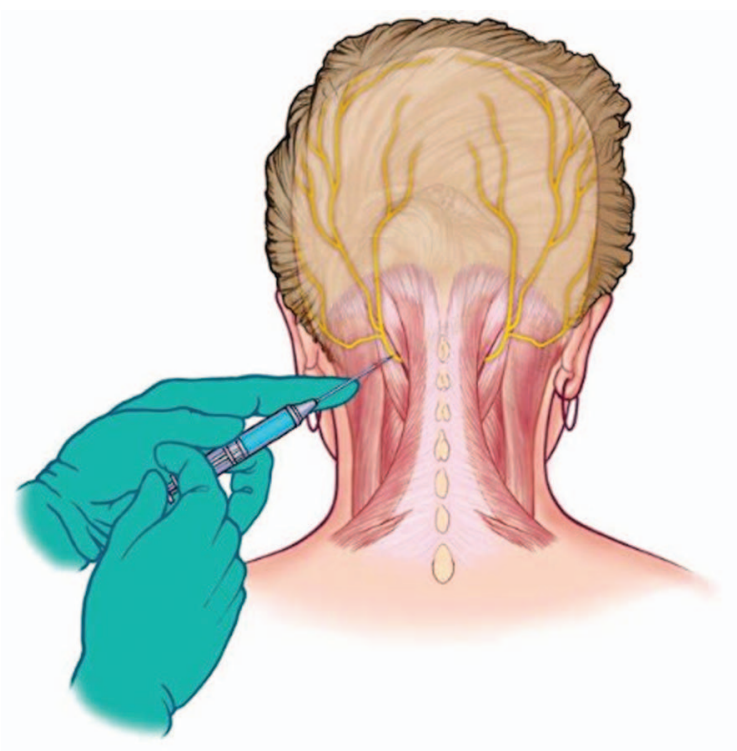

\section{Methods}

\section{Study Design}

This retrospective cohort study was undertaken at the Mayo Clinic in Arizona. An ethical exemption review was obtained from the Mayo Clinic Institutional Review Board before the study commenced. Patients were identified through a search of the electronic medical record (EMR), and we isolated those who had a GON block procedure performed between January 2009 and August 2014. The records included patients treated at the main hospital campus (neurology and pain clinics) in addition to 4 outpatient primary care practices. In total, 732 patients were identified. Inclusion criteria were (1) age 18 to 100 years; (2) migraine headache as defined by the ICHD-3 (beta); (3) received at least 1 GON block; and (4) attended at least 1 follow-up clinic visit. Exclusion criteria were (1) inadequate documentation regarding follow-up and treatment response, (2) concurrent administration of botulinum toxin injection, and (3) headache did not meet diagnostic criteria. All patients included had a diagnosis of migraine (with or without aura) based on the ICHD-3 (beta) guidelines (Figure 2). ${ }^{3}$

\section{Data Collection}

We performed a detailed chart review. We used methodologic strategies as outlined by Gilbert et $\mathrm{al}^{20}$ and Kaji et $\mathrm{al}^{21}$ to help ensure reliability of data abstraction. One researcher (SMA) was assigned the role of chart abstractor, with an aim to minimize interperson variability in data interpretation and documentation. Before starting the data collection phase, the research team held a meeting to clearly delineate the variables that were to be collected and to develop a standardized form into which the data were inserted. Patient demographics including age, sex, referral source, and the department performing the procedure were collected. Procedures were performed by trained groups within the departments of neurology, pain medicine, and primary care. We documented previous treatment regimens (medication-based and nonmedication-based), total number of GON blocks, medication types and doses used, changes in pain scores, and side effects. Several scheduled interim meetings were held to ensure that the quality of the research objective and data collection was being met. A second researcher ( $M M)$ repeated the chart review on a subset of patients $(n=50)$ to ensure a 
reliable data collection technique without significant interperson variability.

\section{Pain Scores}

All patients met criteria for diagnosis of migraine headache based on the ICHD-3 (beta) guidelines, and the majority ( $91 \%$ of the cohort; $\mathrm{n}=500$ ) were in acute pain at the time of their occipital nerve block. The remaining patients had onset of acute migraine-related pain within the 3 days before the procedure. This delay was related to referral and appointment waiting times to see the appropriate specialist. Patients rated their headache-related pain (episodic) before and after the procedure using the 11-point Numeric Pain Rating Scale (NPRS), which ranges from 0 (pain free) to 10 (worst pain imaginable). ${ }^{22,23}$ Pain scores were collected during that same visit immediately before the occipital nerve block procedure. Pain scores were collected again during a follow-up visit, the timing of which ranged between 1 and 4 weeks after the procedure. Change is baseline pain reflected an alteration in pain associated with migraine attacks. Improvement was quantified based on the percentage change from baseline pain score, allowing us to define responses as "minimal" (<30\% NPRS point reduction); "moderate" (31$50 \%$ NPRS point reduction), or "significant" ( $>50 \%$ NPRS point reduction). These categories are derived from several analytic studies that determined a data-derived value of change in the NPRS that best represents a clinically significant improvement in symptoms. ${ }^{24,25}$ These studies, measured using a standard 7-point patient global impression of change, demonstrated a reduction of 2 points, or $30 \%$, as clinically important. ${ }^{24,26}$

\section{Data Analysis}

Basic descriptive statistics, frequency (percentage) for categorical data, and mean \pm standard deviation for continuous data were tabulated for patient demographic characteristics. The Pearson $\chi^{2}$ test and Fisher exact test were used to compare GON block responses among patients with different characteristics. Univariate and multivariate logistic models and Forrest plots were used to determine and display predictors for good responders. To calculate the number needed to treat (NNT), we compared the 2 groups, that is, the event rate in the "treatment group" versus that in the "control group," where the treatment group had $\geq 2$ GON blocks. P values $<.05$ were treated as statistically significant. All analyses were performed using either SAS software version 9.4 (SAS Institute, Inc., Cary, NC) or SPSS version 22.0.0.0 (IBM Corp., Chicago, IL).

\section{Results}

\section{Baseline Patient Demographics}

We assessed 732 patients for inclusion within this study; of these, 562 patients $(76.8 \%)$ met inclusion criteria. We excluded from analysis 170 patients (23.2\%): 21 patients did not meet criteria for migraine diagnosis based on the ICHD-3 (beta) guidelines; 100 did not attend a follow-up visit; 27 did not have adequate documentation of treatment response in the EMR; and 22 patients received botulinum toxin injections during the same encounter. Demographic and clinical characteristics are outlined in Table 1. A total of 319 patients $(56.7 \%)$ had $>1$ GON block, with the average number being 2.34 . The neurology department performed $61 \%$ of the procedures, primary care performed $22 \%$, and pain medicine and neurosurgery performed the remainder. A combina-

Table 1. Baseline Patient Demographics and Clinical Characteristics

\begin{tabular}{lc}
\hline Characteristics & $\begin{array}{c}\text { Patients } \\
(\mathrm{n}=592)\end{array}$ \\
\hline Sex & \\
$\quad$ Female & $423(75.00)$ \\
$\quad$ Male & $139(25.00)$ \\
Age (years) & \\
$\quad<65$ & $329(59.00)$ \\
$\geq 65$ & $233(41.00)$ \\
Previous treatment & \\
Medications (acute and/or prophylactic) & $323(57.00)$ \\
Acupuncture/physical therapy & $1(0.18)$ \\
Combination treatment* & $194(35.00)$ \\
No treatment & $44(8.00)$ \\
Anesthetic & \\
$0.25 \%$ Bupivacaine & $138(25.00)$ \\
$0.5 \%$ Bupivacaine & $369(66.00)$ \\
$1 \%$ Lidocaine & $20(4.00)$ \\
Combination anesthetic & $35(5.00)$ \\
No. of GON block procedures & \\
1 & $228(41.00)$ \\
$\geq 2$ & $334(59.00)$ \\
\hline
\end{tabular}

Data are no. (\%).

${ }^{*}$ Combination treatment refers to the use of both medications and other therapies (e.g., botulinum toxin, acupuncture, physical therapy).

GON, greater occipital nerve. 
Table 2. Treatment Response Across the Different Patient Categories

\begin{tabular}{|c|c|c|c|c|}
\hline \multirow[b]{2}{*}{ Variable } & \multicolumn{3}{|c|}{ Response } & \multirow[b]{2}{*}{$P$ Value } \\
\hline & Minimal (n = 104) & Moderate $(n=130)$ & Significant $(\mathrm{n}=328)$ & \\
\hline Sex & & & & .38 \\
\hline Female & $81(19)$ & $92(22)$ & $250(59)$ & \\
\hline Male & $23(17)$ & $38(27)$ & $78(56)$ & \\
\hline Age (years) & & & & .03 \\
\hline$<65$ & $49(15)$ & $78(24)$ & $202(61)$ & \\
\hline$\geq 65$ & $55(24)$ & $52(22)$ & $126(54)$ & \\
\hline Previous treatment & & & & .52 \\
\hline Medications & $62(19)$ & $71(22)$ & $190(59)$ & \\
\hline Acupuncture/physical therapy & $0(0)$ & $0(0)$ & $1(100)$ & \\
\hline Combination treatment* & $33(17)$ & $53(27)$ & $108(56)$ & \\
\hline No treatment & $9(20)$ & $6(14)$ & $29(66)$ & \\
\hline Previous treatment & & & & .30 \\
\hline None & $9(20)$ & $6(14)$ & $29(66)$ & \\
\hline Medications and combination treatment ${ }^{*}$ & $95(18)$ & $124(24)$ & $299(58)$ & \\
\hline \multicolumn{5}{|l|}{ Anesthetic } \\
\hline Concentration & & & & .92 \\
\hline $0.25 \%$ Bupivacaine & $28(20)$ & $33(24)$ & $77(56)$ & \\
\hline $0.5 \%$ Bupivacaine & $67(18)$ & $86(23)$ & $216(59)$ & \\
\hline 1\% Lidocaine & $2(10)$ & $4(20)$ & $14(70)$ & \\
\hline Combination anesthetic & $7(20)$ & $7(20)$ & $21(60)$ & \\
\hline Dose (milliliters) & & & & .86 \\
\hline $0.25 \%$ bupivacaine & $1.58 \pm 2.84$ & $1.45 \pm 2.82$ & $1.56 \pm 3.10$ & \\
\hline $0.5 \%$ bupivacaine & $1.68 \pm 1.21$ & $1.76 \pm 1.20$ & $1.74 \pm 1.18$ & \\
\hline $1 \%$ lidocaine & $1.0 \pm 3.9$ & $1.3 \pm 1.9$ & $1.7 \pm 1.3$ & \\
\hline No. of GON block procedures & & & & $<.001$ \\
\hline 1 & $79(35)$ & $67(29)$ & $82(36)$ & \\
\hline$\geq 2$ & $25(7)$ & $63(19)$ & $246(74)$ & \\
\hline Use \% lidocaine & & & & .77 \\
\hline No & $95(19)$ & $119(23)$ & $294(58)$ & \\
\hline Yes & $9(17)$ & $11(20)$ & $34(63)$ & \\
\hline
\end{tabular}

Data are no. (\%) unless otherwise indicated.

${ }^{*}$ Combination treatment refers to the use of both medications (acute and prophylactic) in addition to other therapies (e.g., botulinum toxin, acupuncture, physical therapy).

$P<.05$ was considered significant (in bold).

GON, greater occipital nerve.

tion of steroid and local anesthetic was used in $99.3 \%$ of patients; $0.7 \%$ received local anesthetic alone.

\section{Clinical Efficacy}

Of the cohort who received GON block, $82 \%$ quantified their response to treatment as moderate or significant, indicating a reduction in their baseline pain score of $\geq 30 \%$. A total of 328 patients $(58 \%)$ had a significant response, indicating a reduction in baseline pain score of $\geq 50 \%$. The data set was analyzed to compare treatment responses between the groups, and a multivariate logistic model was used to assess for response predictors.
As shown in Table 2, $61 \%$ of patients aged $<65$ years had a significant response to treatment compared with $54 \%$ of patients aged $\geq 65$ years $(P=$ .03). Among patients who underwent $\geq 2$ GON blocks, $74 \%$ had a significant response, compared with $36 \%$ of patients who underwent 1 GOB block alone $(P<.001)$, as shown in Figure 3.

Across the different age categories, patients who underwent $\geq 2$ GON block procedures responded equally well, with an odds ratio of 4.9 to $5.0(P<.001)$. Outcomes did not differ based on anesthetic concentration or volume of administration across the different patient groups. Aver- 
Figure 2. Study flow diagram.

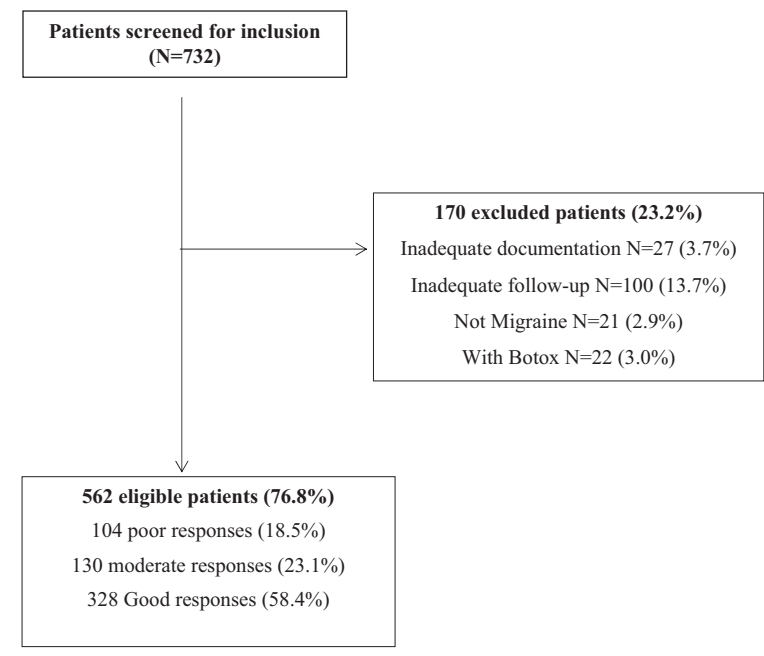

age volumes of administration for the different anesthetic concentrations (doses) is listed in Table 3 .

We divided patients based on previous treatment regimens, different medication combinations, and department performing the procedure and found no statistically significant difference in response across the groups.

\section{Number Needed to Treat}

No control group was used in this study, given its retrospective design. We were, however, able to compare response rates in those patients treated with 1 GON block with those treated with $\geq 2$ GON blocks. In this case, the NNT was calculated at 2.6.
Table 3. Concentration and Volume of Anesthetic Used

\begin{tabular}{ll}
\hline Anesthetic & Volume of Injection (milliliters) \\
\hline $0.25 \%$ Bupivacaine & $6.30(1.50-20.00)$ \\
$0.5 \%$ Bupivacaine & $2.50(0.25-5.00)$ \\
$1 \%$ Lidocaine & 2.40 \\
\hline
\end{tabular}

Data are mean (range).

\section{Safety}

Details of the GON block procedure were outlined in a procedural note contained within the EMR. These were reviewed to assess for adverse procedural reactions or complications. Few adverse reactions were reported in the period immediately following the procedure: 10 patients developed vasovagal symptoms that responded to conservative treatment measures, and 25 patients described "burning" at the injection site. No reports of lasting complications or side effects were found within the follow-up visit documentation.

\section{Discussion}

The findings from this large, retrospective cohort study suggest that GON block is an effective treatment for acute management of migraine headache. A large percentage of our cohort reported a reduction in both episodic migraine related pain of $>30 \%$ (from their baseline NPRS value) following treatment with GON block. These results were consistent regardless of sex, age, prior treatments, single or multiple GON blocks, or specialty performing the procedure. A significant correlation was found between receiving $\geq 2$ GON blocks and

Figure 3. Response rates across the different numbered categories for greater occipital nerve block.

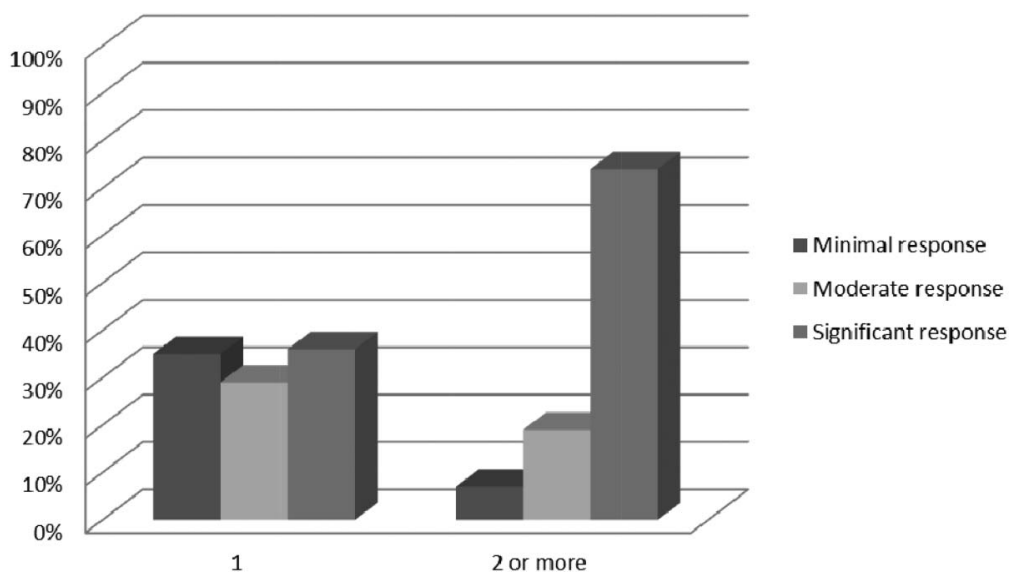


reduction in pain score $(P<.001)$. In fact, when we compared the rate of response in patients who received 1 GON block with those who received $\geq 2$, the NNT was calculated as 2.6. We hypothesize that repeated peripheral nerve blockade and associated central neuromodulation may increase pain thresholds and thereby improve desensitization. This may have been of particular importance in this older patient cohort. It is important to consider that those patients who obtained good results with 1 procedure are more likely to return for repeated procedures. Another consideration was the duration of time between repeated GON blocks; a short window of time could account for better results. We were unable to obtain this information from the EMR and, as such, cannot account for this as a possible confounder.

No relationship was found between previous treatment regimens (medications, physical therapy, botulinum toxin, acupuncture, or a combination of therapies) and response to GON block $(P=.52)$, suggesting that efficacy of the procedure is not affected by the treatment history. Treatment response was similar across the different age and sex groups. It is worth noting that the mean age of this study population was older than patients in typical migraine studies, which may have had a positive effect on our results as a result of possible cervicogenic triggers/components that may also be addressed with GON blockade.

The majority of the literature pertaining to the use of GON block in the treatment of migraine headache comes from observational studies, ${ }^{8,11-13}$ most of which showed improvement in headache symptoms, intensity, and frequency. Some studies looked at the efficacy of GON block in the treatment of other headache syndromes, including cluster headache, chronic daily headache, and cervicogenic headache, along with separate pathologies including trigeminal neuralgia and post-dural puncture headache. ${ }^{27}$ The first double-blind, randomized controlled trial was performed by Piovesan et $\mathrm{al}^{16}$ in 2001; they included 37 patients and compared GON block to placebo as preventive management of migraine, and in a crossover design performed a sham procedure or GON block. Although they found no difference in the number or duration of migraine episodes between the procedures, the group that received GON block experienced a significant reduction in migraine intensity during the 60-day follow-up period. Ashkenazi and
Young ${ }^{10}$ performed a prospective, noncontrolled study to look at the effect of GON block on head pain and allodynia in a cohort of 19 patients with migraine. Both local anesthetic and steroids were used. They noted a significant improvement after the procedure, with $90 \%$ of the group documenting relief from head pain. The most recent randomized controlled trial was published in 2016 by Cuadrado et $\mathrm{al}^{19}$ and included 36 patients with chronic migraine treated with bilateral GON block with bupivacaine $(\mathrm{n}=18)$ or a sham procedure with normal saline $(\mathrm{n}=18)$. Anesthetic block was superior to placebo in reducing the frequency of headaches during the week after injection, along with an increase in pressure pain thresholds in various territories.

Prior studies have suggested that different GON block treatment regimens should be compared in order to assess what effect, if any, the addition of steroid or different doses/types of local anesthetics would have on efficacy results. We found no difference in response despite the use of various combinations and doses of local anesthetic. As previously mentioned, only 4 patients within our cohort did not receive corticosteroid as part of their GON block, and therefore we are unable to comment on treatment response using anesthetic alone. We also are unable to draw any comparisons between the 2 procedural strategies.

The scope of this study was to assess, in a retrospective manner, the response to treatment with GON block in a cohort of patients with episodic migraine headache. Change in baseline pain scores reflected a spectrum of pain relief for these patients, including relief of short-term pain, pain from acute attacks, and continuous pain. The use of a validated pain score allowed us to quantify response to treatment in a way that can be interpreted and applied clinically. This study has some limitations we would like to highlight. We were limited by the retrospective design, which precluded us from using a control group. We need to consider the confounding effect that the placebo response may have on our results and mention that innate selection bias existed because of the retrospective design and inclusion criteria. Furthermore, we were unable to divide patients based on the goal of treatment, be it acute or prophylactic management, and as such we did not perform subgroup analysis based on these criteria. We believe the major strength of our study is derived from its large sample size, which, to our knowledge, 
greatly exceeds that of any previously published article on this topic. We believe this adds power and applicability to our findings, despite the retrospective design.

The suggested biological mechanism by which GON block works to treat the symptoms of migraine relates to modulation of afferent signals to the trigeminal nucleus caudalis, which bridges the gap between sensory regions of the ophthalmic branch of the trigeminal nerve and the greater occipital nerve. Injecting this region with local anesthetic and corticosteroids decreases sensory input to the trigeminal nucleus caudalis. Overall, our findings are very positive in terms of the efficacy of GON block as a treatment option for migraine headache. Our figures for response reflect closely some of the studies mentioned earlier, including Caputi and Firetto, ${ }^{12}$ who described headache improvement in $85 \%$ of their cohort, and Ashkenazi and Young, ${ }^{10}$ who documented decrease in head pain in $90 \%$ of their patients after GON block. Both of these studies had much smaller sample sizes: 27 and 19 patients, respectively.

\section{Conclusion}

In a large, retrospective cohort study of patients with migraine headache, GON block used as the sole treatment strategy or as an adjunct to other therapeutic modalities resulted in high rates of clinical response. Large placebo-controlled clinical trials are needed to confirm our findings, along with those from several smaller observational studies and randomized trials. Further data would help to solidify the use of GON block in the treatment of migraine headache and potentially assist with its inclusion within future treatment guidelines Figure 2.

The authors acknowledge JoAnn McBroom for her assistance with formatting this manuscript in preparation for publication.

To see this article online, please go to: bttp://jabfm.org/content/ 31/2/211.full.

\section{References}

1. Silberstein SD, Holland S, Freitag F, Dodick DW, Argoff C, Ashman E; Quality Standards Subcommittee of the American Academy of Neurology and the American Headache Society. Evidence-based guideline update: pharmacologic treatment for episodic migraine prevention in adults: report of the Quality
Standards Subcommittee of the American Academy of Neurology and the American Headache Society. Neurology 2012;78:1337-45.

2. Silberstein SD, Goadsby PJ. Migraine: preventive treatment. Cephalalgia 2002;22:491-512.

3. Headache Classification Committee of the International Headache Society. The International Classification of Headache Disorders, 3rd edition (beta version). Cephalalgia 2013;33:629-808.

4. Naumann M, So Y, Argoff CE, Childers MK, et al; Therapeutics and Technology Assessment Subcommittee of the American Academy of Neurology. Assessment: botulinum neurotoxin in the treatment of autonomic disorders and pain (an evidence-based review): report of the Therapeutics and Technology Assessment Subcommittee of the American Academy of Neurology. Neurology 2008;70:1707-14.

5. Guyuron B, Varghai A, Michelow BJ, Thomas T, Davis J. Corrugator supercilii muscle resection and migraine headaches. Plast Reconstr Surg 2000;106: 429-34, discussion 35-7.

6. Reed KL, Black SB, Banta CJ 2nd, Will KR. Combined occipital and supraorbital neurostimulation for the treatment of chronic migraine headaches: initial experience. Cephalalgia 2010;30:260-71.

7. Ashkenazi A, Levin M. Greater occipital nerve block for migraine and other headaches: is it useful? Curr Pain Headache Rep 2007;11:231-5.

8. Afridi SK, Shields KG, Bhola R, Goadsby PJ. Greater occipital nerve injection in primary headache syndromes-prolonged effects from a single injection. Pain 2006;122(1-2):126-9.

9. Ashkenazi A, Matro R, Shaw JW, Abbas MA, Silberstein SD. Greater occipital nerve block using local anaesthetics alone or with triamcinolone for transformed migraine: a randomised comparative study. J Neurol Neurosurg Psychiatry 2008;79:415-7.

10. Ashkenazi A, Young WB. The effects of greater occipital nerve block and trigger point injection on brush allodynia and pain in migraine. Headache 2005;45:350-4.

11. Bovim G, Sand T. Cervicogenic headache, migraine without aura and tension-type headache. Diagnostic blockade of greater occipital and supra-orbital nerves. Pain 1992;51:43-8.

12. Caputi CA, Firetto V. Therapeutic blockade of greater occipital and supraorbital nerves in migraine patients. Headache 1997;37:174-9.

13. Gawel MJ, Rothbart PJ. Occipital nerve block in the management of headache and cervical pain. Cephalalgia 1992;12:9-13.

14. Inan N, Ceyhan A, Inan L, Kavaklioglu O, Alptekin A, Unal N. C2/C3 nerve blocks and greater occipital nerve block in cervicogenic headache treatment. Funct Neurol 2001;16:239-43.

15. Leinisch-Dahlke E, Jurgens T, Bogdahn U, Jakob W, May A. Greater occipital nerve block is ineffec- 
tive in chronic tension type headache. Cephalalgia 2005;25:704-8.

16. Piovesan EJ, Werneck LC, Kowacs PA, Tatsui CE, Lange MC, Vincent M. Anesthetic blockade of the greater occipital nerve in migraine prophylaxis [article in Portuguese]. Arq Neuropsiquiatr 2001;59(3A):545-51.

17. Dilli E, Halker R, Vargas B, et al. Occipital nerve block for the short-term preventive treatment of migraine: a randomized, double-blinded, placebocontrolled study. Cephalalgia 2015;35:959-68.

18. Inan L, Inan N, Karadaş Ö, et al. Greater occipital nerve blockade for the treatment of chronic migraine: a randomized, multicenter, double-blind, and placebocontrolled study. Acta Neurol Scand 2015;132:270-7.

19. Cuadrado ML, Aledo-Serrano Á, Navarro P, et al. Short-term effects of greater occipital nerve blocks in chronic migraine: a double-blind, randomised, placebo-controlled clinical trial. Cephalalgia 2017; 37:864-72.

20. Gilbert EH, Lowenstein SR, Koziol-McLain J, Barta DC, Steiner J. Chart reviews in emergency medicine research: where are the methods? Ann Emerg Med 1996;27:305-8.

21. Kaji AH, Schriger D, Green S. Looking through the retrospectoscope: reducing bias in emergency medicine chart review studies. Ann Emerg Med 2014;64: 292-8.
22. Downie $W$, Leatham $P$, Rhind V, Wright V, Branco J, Anderson J. Studies with pain rating scales. Ann Rheum Dis 1978;37:378-81.

23. Price DD, Bush FM, Long S, Harkins SW. A comparison of pain measurement characteristics of mechanical visual analogue and simple numerical rating scales. Pain 1994;56:217-26.

24. Farrar JT, Young JP, LaMoreaux L, Werth JL, Poole RM. Clinical importance of changes in chronic pain intensity measured on an 11-point numerical pain rating scale. Pain 2001;94:149-58.

25. Childs JD, Piva SR, Fritz JM. Responsiveness of the numeric pain rating scale in patients with low back pain. Spine 2005;30:1331-4.

26. Hawker GA, Mian S, Kendzerska T, French M. Measures of adult pain: Visual Analog Scale for Pain (VAS Pain), Numeric Rating Scale for Pain (NRS Pain), McGill Pain Questionnaire (MPQ), Short-Form McGill Pain Questionnaire (SFMPQ), Chronic Pain Grade Scale (CPGS), Short Form-36 Bodily Pain Scale (SF-36 BPS), and Measure of Intermittent and Constant Osteoarthritis Pain (ICOAP). Arthritis Care Res (Hoboken) 2011;63(Suppl 11):S240-52.

27. Ashkenazi A, Blumenfeld A, Napchan U, et al. Peripheral nerve blocks and trigger point injections in headache management - a systematic review and suggestions for future research. Headache 2010;50: 943-52. 